\title{
Modification of Frozen Elephant Trunk Technique for Acute Type-A Aortic Dissection - A Case Report
}

\author{
SAIKAT DAS GUPTA ${ }^{1}$, P K CHANDA ${ }^{1}$, D K ADHIKARY ${ }^{2}$ \\ ${ }^{1}$ Department of Cardiac Surgery, Square Hospitals Limited, Dhaka, Bangladesh, ${ }^{2}$ Department of Cardiology, Bangabandhu \\ Sheikh Mujib Medical University, Dhaka, Bangladesh. \\ Address of Correspondence: Dr. Saikat DasGupta, Junior Consultant, Department of Cardiac Surgery, Square Hospitals \\ Limited, West Panthapath, Dhaka 1205, Bangladesh, E-mail: saikatdasgupta@ymail.com
}

\begin{abstract}
:
Acute type-A aortic dissection (ATAAD) is a dreadfully fatal cardiovascular emergency. The mortality rate among medically treated patients is $50 \%$ within two days. Surgery is the gold standard and urgent surgical treatment is indicated for all patients, except for those who are moribund or severely comorbid. However, early mortality after operation remains high (9-25\%). We hereby present a case of 22 years young, male, Marfan patient with a family history of ATAAD treated successfully by us at Square Hospital Ltd by modified Bentall's procedure with total arch replacement with Frozen Elephant Trunk (FET) technique.
\end{abstract}

University Heart Journal 2022; 18(1): 65-70

\section{Introduction:}

Acute aortic dissection is an unexpected life-threatening condition of the aorta that accompanies significant morbidity and mortality. ${ }^{1}$ Ascending aortic involvement regardless of extension into the aortic arch or the descending aorta is classified as Stanford type-A dissections. ${ }^{2,3}$ A sudden, intense "ripping" or "tearing" chest pain usually refers to ATAAD. ${ }^{3}$ The pain is usually present retrosternal or substernal, with distal or proximal spread as the dissection evolves. The patient typically seeks immediate hospital care or in rare cases, presents with less severe symptoms and is revealed in a subacute phase, or leads to sudden death. ${ }^{3}$ In one-third of the patients, the symptoms are associated with organ mal-perfusion due to diminished flow in end-artery organs. ${ }^{4}$ Clinically lower extremity mal-perfusion and pulse deficit are easily detected. Occasionally, cerebral ischemia or paraplegia is the first presenting symptom of acute aortic dissection. ${ }^{2}$

ECG may show signs of cardiac ischemia or non-specific ST and T-wave alterations, as dissection may involve coronary Ostia upsetting the coronary flow. ${ }^{5}$ Chest X-ray may show a wide mediastinum, ${ }^{6}$ but precise diagnosis is done by CT scan of the aorta. ${ }^{7}$ CT aorta shows the true and the false lumen of the aorta, identify entry tear, delineates the extent of the dissection and the involvement of the branch arteries including the arch vessels. The sensitivity and specificity of CT are excellent ${ }^{7}$; but in patients with renal insufficiency or in persons allergic to iodinated dye, the role of CT angiography is disputed and other modalities like magnetic resonance imaging (MRI) can be considered instead. ${ }^{8}$ Transthoracic echocardiography (TTE) reveals the ventricular function, valves, and aortic root and shows aortic dilatation, pericardial effusion, and tamponade, but TTE is associated with poor visibility in the obese, in patients with chest deformities, and in patients on mechanical ventilation. ${ }^{9}$ Detailed information like aortic valve regurgitation can be obtained with transesophageal echocardiography (TEE), and often primary intimal tear in the aortic root can be identified. ${ }^{9}$ Coronary angiography (CAG) is not indicated as a primary diagnostic method for ATAAD, but in suspected acute coronary syndrome (ACS) at times significant coronary artery disease is detected (around 30\%) at CAG of ATAAD patients, that may necessitate treatment and affect outcomes. ${ }^{10}$

\section{Case Report:}

Mr. X, 22 years of age, a male patient got admitted to the emergency room with the chief complaints of sudden severe chest pain for the last four hours. The pain was radiating to both sides of the shoulder, and was associated with exertional shortness of breath, not relieved by rest or drugs, and was not associated with nausea or vomiting. He was a known patient of Marfan Syndrome with a family 


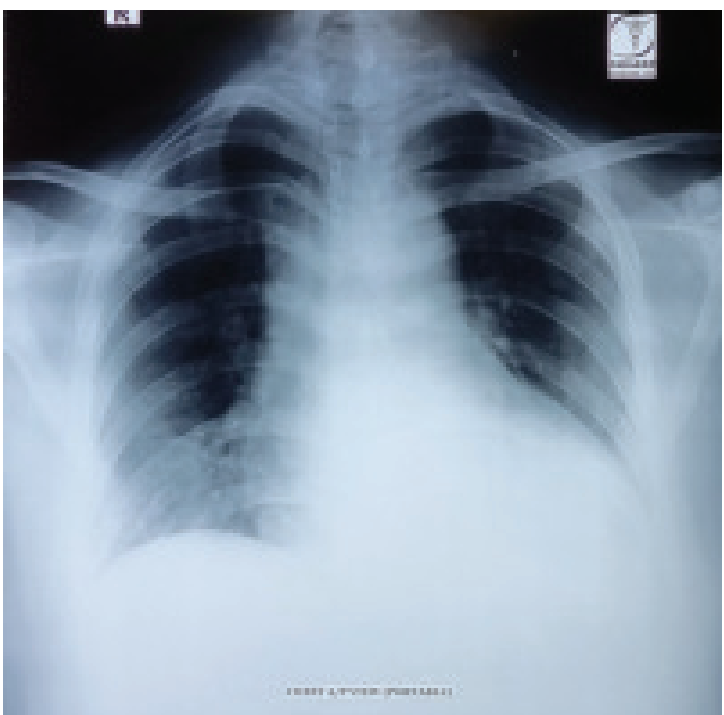

Fig.-1: Pre-operative $X$-ray chest $P A$ view

history of ATAAD. The patient has no history of Diabetes, Chronic Kidney Disease, Cerebrovascular Disease, Chronic Obstructive Pulmonary Disease, Bronchial Asthma, or any major surgery.

His pre-operative BP was 170/70 $\mathrm{mm}$ of $\mathrm{Hg}$ and his heart rate was $97 \mathrm{~b} / \mathrm{min}$. All his pre-operative biochemical reports were normal except serum bilirubin was $1.7 \mathrm{mg} / \mathrm{dl}$ and CRP was $81.3 \mathrm{mg} / \mathrm{L}$. His NT-proBNP was $1357 \mathrm{pg} / \mathrm{ml}$ and s. creatinine was $0.9 \mathrm{mg} / \mathrm{dl}$. His BSA was $2.4 \mathrm{~m} 2$, and his Euroscore II for in-hospital mortality was $10.18 \%$.

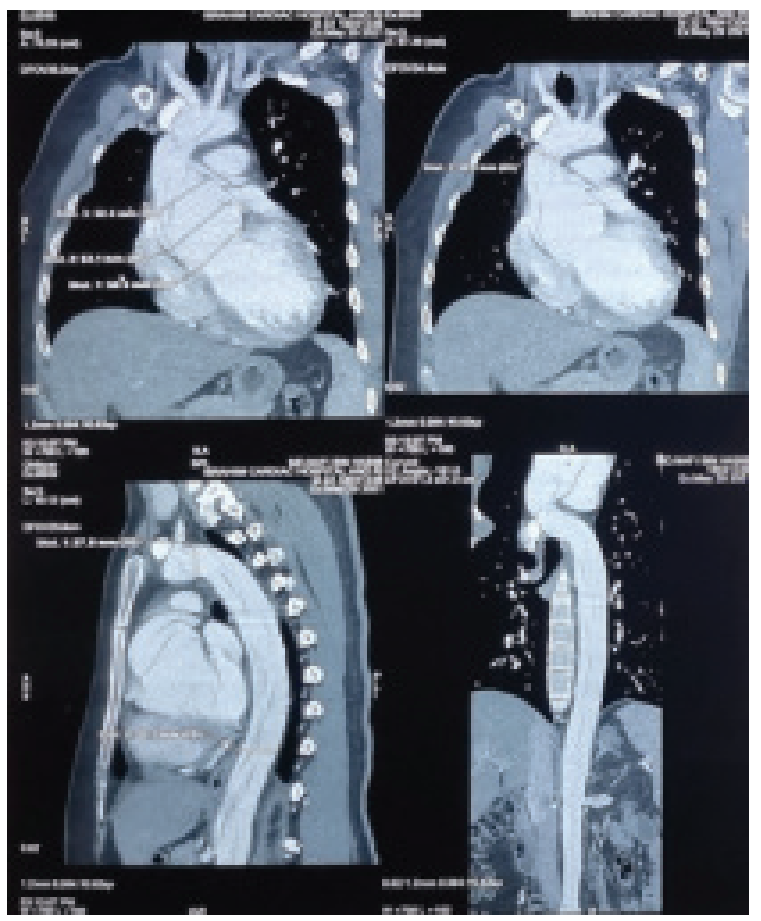

Fig.-2: Pre-operative CT scan of aorta showing acute dissection with extension

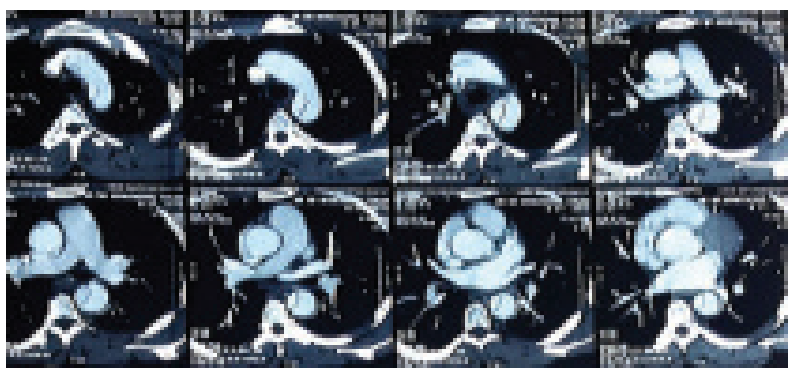

Fig.-3: Pre-operative CT scan of aorta showing acute dissection with true and false lumen

The patient's pre-operative color Doppler echocardiogram showed features of dissecting aortic aneurysm, flap noted from the aortic root with extension to descending thoracic aorta (DTA). Tricuspid aortic valve (AV) with moderate to severe aortic regurgitation (AR) was noted. AV annulus $33 \mathrm{~mm}$, sinus $67 \mathrm{~mm}$, and ascending aorta was $47 \mathrm{~mm}$. Good RV systolic function, global LV hypokinesia, with concentric LVH seen. His LVIDD/LVIDS was 70/56 mm with Moderate LV systolic dysfunction (EF-40-45\%) and PASP-was $35 \mathrm{~mm}$ of $\mathrm{Hg}$. No pericardial effusion or intracardiac thrombus was seen.

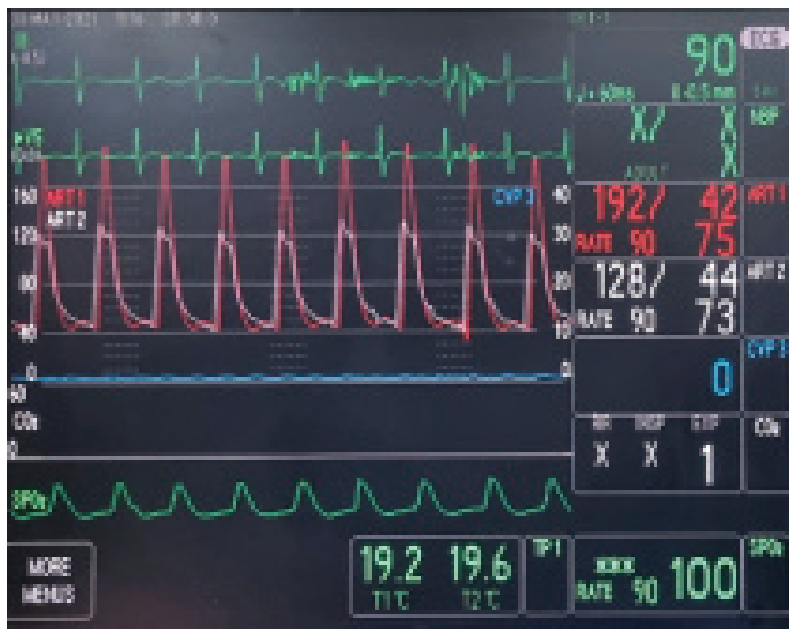

Fig.-4: Gross differences of blood pressure between right radial (red) and left femoral (White) artery

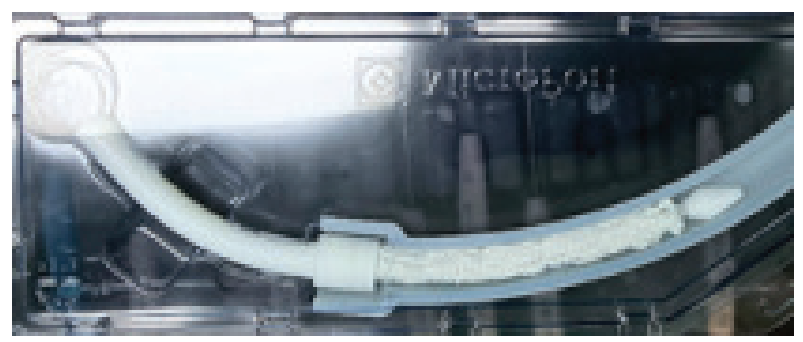

Fig.-5: Frozen elephant trunk prosthesis (CRONUS®) before deployments 


\section{Surgery:}

The patient was operated under all aseptic pre-caution on the subsequent morning of May 2021 on an emergency basis. Central venous line (Right, jugular), right radial and left femoral arterial lines, rectal and nasopharyngeal temperature probes along with NIRS (near-infrared spectroscopy) were established. After median sternotomy, the thymus was dissected and controls were taken around Brachiocephalic / Left common carotid / left subclavian arteries. Pericardiotomy was done and after heparinization and proper ACT, CPB was established with right innominate artery with $10 \mathrm{~mm}$ tube graft and RA cannulation. The patient was cooled to $25^{\circ} \mathrm{C}$. An Aortic $\mathrm{X}$ clamp was applied and aortotomy was done followed by delivery of del-Nido cardioplegia. The heart was arrested at diastole. Dissection found extending up to sinus of Valsalva and aortic valve was found severely incompetent. To reduce $\mathrm{CPB}$ and $\mathrm{ACC}$ time we did not attempt valve repair; rather modified Bentall's procedure was done with a $25 \mathrm{~mm}$ mechanical valved conduit. TCA started (TCA time 31 minutes) and antegrade cerebral perfusion was established through Brachiocephalic trunk and monitored with NIRS. $22 \mathrm{~mm}$ CRONUS ${ }^{\circledR}$ frozen elephant trunk (FET) prosthesis was deployed at Zone 2, and FET was anastomosed with branched graft (distal anastomosis). Subsequently, sequential replacement of total aortic arch with debranching of LSA, proximal anastomosis, LCC, and innominate artery debranching were done. All the suture lines were checked and bio-glue was applied at the proximal suture line. The aortic crossclamp was released after proper de-airing. Heart weaned from CPB at normal sinus rhythm. Total CPB time was 218 minutes and ACC time was 163 minutes. TEE showed a normally functioning aortic valve. De-cannulation done. After achieving hemostasis chest wound was closed in

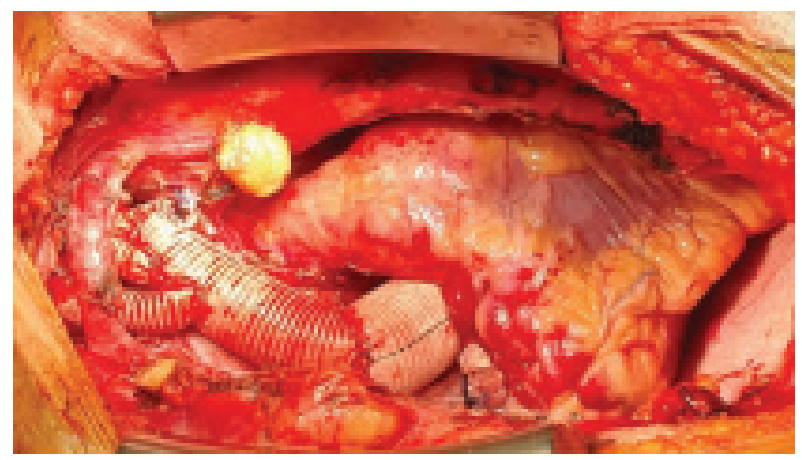

Fig.-6: Reconstructed aorta with branches after completion of the procedure layers keeping chest drain tubes and RV pacing wire in situ.

The patient was shifted to ICU with minimum inotrope support and the patient was extubated on the subsequent morning. His post-operative chest drainage was minimum. The patient's in-hospital course was eventless and once had a mild decrease of hemoglobin on the $2 \mathrm{nd}$ Postoperative day which was normalized on the 4 th postoperative day. The patient's postoperative color Doppler echocardiogram showed a normally functioning aortic valve and the normal flow inside the aortic conduit. No valvular or paravalvular leakage seen. Good RV systolic function, global LV hypokinesia was seen. Moderate LV systolic dysfunction (EF-45\%), Concentric LVH, PASP$30 \mathrm{~mm}$ of $\mathrm{Hg}$. No pericardial effusion or intra-cardiac thrombus was seen. The patient was discharged on the 8th postoperative day with $5 \mathrm{mg}$ warfarin and an INR of 2.14.

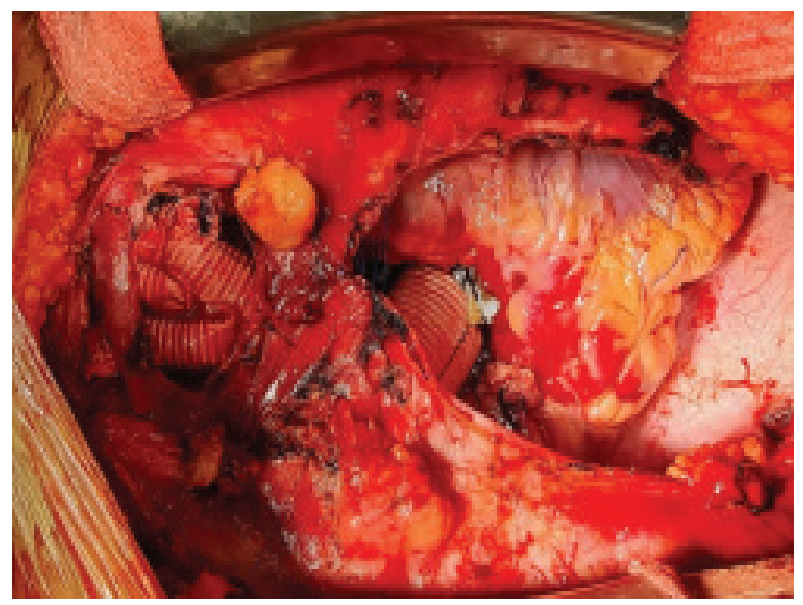

Fig.-7: Just before sternal closure (aorta is covered with thymic tissue)

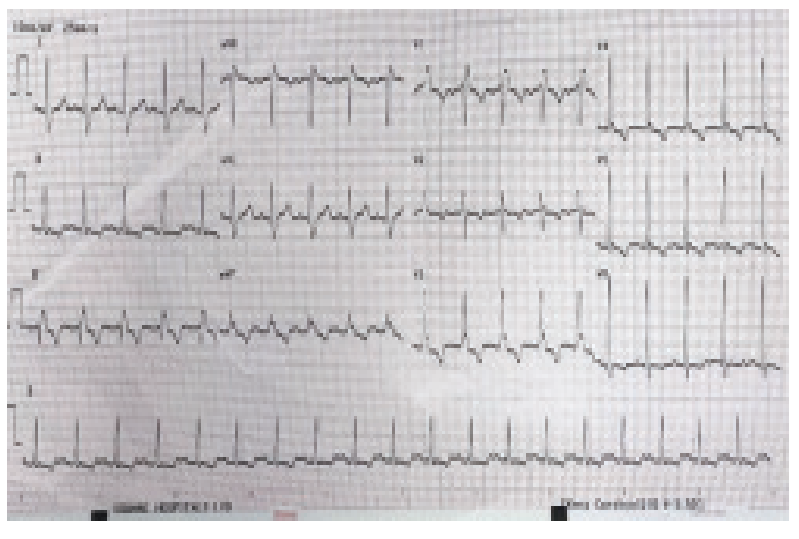

Fig.-8: Post-operative ECG showing RBBB and left ventricular hypertrophy 


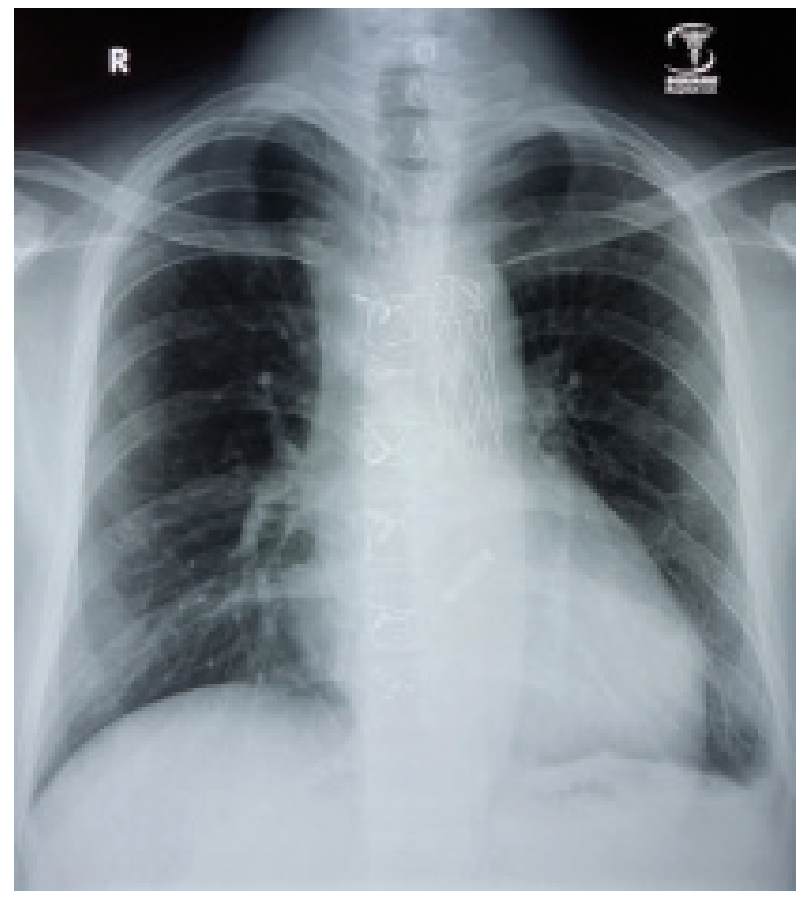

Fig.-9: Post-operative X-ray chest showing FET and mechanical valve (aortic) in-situ

\section{Discussion:}

Based on its natural course, the estimated overall fatality rate of ATAAD is $73 \%$, and $1849 \%$ of patients with ATAAD succumbs to death before hospital admission. ${ }^{11,12}$ In-hospital mortality rate is $58 \%$, in admitted patients who refuse surgical treatment or are not offered surgery. ${ }^{6}$ In type A acute aortic dissection medical therapy to control pain and hypertension is essential in all patients. Betablockers can reduce blood pressure (systolic 100-120 $\mathrm{mmHg}$ ), reduce stress on the aortic wall; heart rate (approximately $60 \mathrm{bpm}$ ), and protect the myocardium against ischemia. ${ }^{13}$ Otherwise, vasodilators like calcium channel blockers (nicardipine or diltiazem) or nitroglycerin are useful in reducing hypertension in emergency situations. In our patient preoperative BP was 170/70 mm of $\mathrm{Hg}$ with a regular heart rate of $97 \mathrm{~b} / \mathrm{min}$. We started intravenous Nitroglycerine (GTN) 5 microgram $/ \mathrm{kg} / \mathrm{min}$ along with oral Bisoprolol $5 \mathrm{mg} 12$ hourly, Amlodipine 5 mg 12 hourly, and Prazosin 2 mg orally 8 hourly and by the next morning BP came down to $130 / 70 \mathrm{~mm}$ of $\mathrm{Hg}$. However, mortality in medically treated patients increases by $1 \%$ to $2 \%$ for every hour after the onset of symptoms, reaches 30 68\% within two days, and reaches 49 73\% within the first two weeks, ${ }^{1,14}$ thus clearly confirming surgery being the primary treatment of choice. Emergency open surgical repair is the standard of care in ATAAD ${ }^{15}$.
Unless prompt surgical repair is performed, the patient usually succumbs to death due to complications like rupture of the aorta, myocardial ischemia, pericardial tamponade, acute aortic regurgitation, end-organ malperfusion, or acute heart failure. ${ }^{2}$ Although surgical results are still unsatisfactory, long-term outcomes after the operation are obviously better, compared with medical therapy alone.

De Bakey et al. defined the basic principle of repair comprising (1) excision of the intimal tear, (2) obliteration of entry into the false lumen, and (3) aortic reconstitution with interposition tube synthetic graft with or without reimplantation of the coronary arteries. ${ }^{17}$ When the primary intimal tear is located in the aortic arch, both the ascending aorta and aortic arch are replaced (TAR). ${ }^{18}$ TAR (Total arch replacement) comprises deployment of the frozen elephant trunk (FET) from the zone- 0 of the aorta into the descending aorta, ascending aortic replacement, and reconstruction of arch vessel. If the aortic valve is normal or near-normal, a valve-sparing operation may be an option. ${ }^{19}$ Even though, valve-sparing procedures increase surgical time compared with composite graft replacement with an artificial valve, but they have the benefit of avoiding anti-coagulants in the long run. In our patient to minimize the operative time, we had to avoid the valve-sparing procedure. In some patients with myocardial ischemia or infarction because of coronary artery dissection or compression by a false lumen, coronary artery bypass grafting may be needed. ${ }^{20}$

In 2003, Karck et al., reported open aortic arch replacement with stent-graft insertion in the descending aorta. ${ }^{21}$ A custom-made stent-graft (Chavan-Haverich ${ }^{\circledR}$, Curative Medical Devices Gmbh, Dresden) was used, and this method was named "frozen elephant trunk (FET)"; this was the first reported series of FET. FET practically eliminates the need for surgical replacement of the descending aorta. ${ }^{22}$ For TAR \& FET Sun et al., ${ }^{28}$ showed sequences of distal anastomosis of LCC, Proximal anastomosis, the release of aortic cross-clamp, LSA, and Brachiocephalic debranching, then after distal anastomosis rewarming usually started. But, from our case to case experience, we found after Zone-2 distal anastomosis \& LCC debranching, it is difficult to go for LSA debranching. So we modified the sequence, distal anastomosis, LSA debranching, proximal anastomosis, cross-clamp release followed by LCC and Brachiocephalic debranching.

After surgery for type A aortic dissection, complications like stroke (2-16\%), has been reported in recent studies. ${ }^{23}$ Renal failures, spinal cord injury, mediastinal bleeding, 
chylothorax, and mediastinitis are other noteworthy complications. Bleeding is an independent predictor of mortality (both early and late) after surgery for ATAAD and is liable for $20 \%$ of in-hospital deaths. ${ }^{19}$ FET grafts are reportedly associated with spinal cord ischemia. The overall early mortality from ATAAD (30-days or inhospital) post-operative patients ranges from $5-24 \%$ in current reports. ${ }^{24}$ Patients who remain clinically stable preoperatively with normal perfusion generally do well after surgery, with low mortality (of around $3 \%$ ). ${ }^{25}$ On the contrary, patients with unstable hemodynamics or preoperative mal-perfusion, have a significant mortality rate $(1840 \%){ }^{26}$ These extended surgeries predictably increase patients' surgical trauma; so a conservative approach can be obtained especially in elderly patients ( $>75$ years) and in those who come with a critical status, as established by prolonged shock/tamponade, brain, and mesenteric ischemia. Younger patients with a minor complication related to acute dissection and those with a complex distal tear or aortic rupture are the best candidates for the FET technique. ${ }^{27}$ At follow-up after one month and sixth months of surgery patient was doing satisfactory daily activities.

\section{Conclusion:}

In patients with type A aortic dissection, the gold standard treatment is surgery. Surgical treatment for aortic pathology is offered at very few centers in our country and by even fewer teams exclusively. It makes the time-consuming transportation to the specific center crucial in some of the cases. Then again, and more commonly, patients usually arrive at the operating center in a more deranged condition due to longer periods of mal-perfusion, ischemia, tamponade, or suboptimal hemodynamic control. Thus, optimal management of ATAAD patients requires surgical adaptation and resourcefulness, typically provided more and more often at dedicated aortic centers. Early diagnosis and prompt referral is the main key to survival in those patients.

\section{Ethical issues:}

The authors have taken appropriate consent from the patient, that his images and other clinical information be reported in the journal. He understands that his name and initials will not be published, and due efforts will be made to conceal his identity, but anonymity cannot be guaranteed.

\section{Acknowledgments:}

The authors would like to acknowledge Dr. Mesbahuddin Dolon, Dr. Jagodananda Roy, Mr. Mohammad Kamal
Uddin, Mr. Tanvir Ahmed for their relentless effort during surgery and data collection.

\section{Disclosure: None}

\section{References:}

1. Jassar AS, Sundt TM, III. How should we manage type A aortiac dissection? Gen Thorac Cardiovasc Surg. 2019:67(1):137-45.

2. Nienaber CA, Clough RE. Management of acute aortic dissection. Lancet 2015;385(9970):800-811.

3. Elsayed RS, Cohen RG, Fleischman F, et al. Acute type A aortic dissection. Cardiol Clin. 2017;35(3):331-45.

4. Czerny M, Schoenhoff F, Etz C, et al. The impact of pre-operative malperfusion on outcome in acute type A aortic dissection: results from the GERAADA Registry. J Am Coll Cardiol. 2015;65(24):2628-35.

5. Evangelista A, Isselbacher EM, Bossone E, et al. Insights from the International Registry of Acute Aortic Dissection: a 20-year experience of collaborative clinical research. Circulation. 2018;137(17):1846-60.

6. Hagan PG, Nienaber CA, Isselbacher EM, et al. The International Registry of Acute Aortic Dissection (IRAD): *new insights into an old disease. JAMA. 2000;283(7):897-903.

7. Rogers IS, Banerji D, Siegel EL, et al. Usefulness of comprehensive cardiothoracic computed tomography in the evaluation of acute undifferentiated chest discomfort in the emergency department (CAPTURE). Am J Cardiol. 2011;107(5):643-50.

8. Wang GX, Hedgire SS, Le TQ, et al. MR angiography can guide ED management of suspected acute aortic dissection. Am J Emerg Med. 2017;35(4):527-30.

9. Meredith EL, Masani ND. Echocardiography in the emergency assessment of acute aortic syndromes. Eur J Echocardiogr. 2009; 10(1):i31-i39.

10. Tsagakis K, Konorza T, Dohle DS, et al. Hybrid operating room concept for combined diagnostics, intervention and surgery in acute type A dissection. Eur J Cardiothorac Surg. 2013;43(2):397404.

11. Howard DP, Banerjee A, Fairhead JF, et al. Population-based study of incidence and outcome of acute aortic dissection and premorbid risk factor control: 10-year results from the Oxford Vascular Study. Circulation 2013;127(20):2031-37.

12. Rylski B, Hoffmann I, Beyersdorf F, et al. Acute aortic dissection type A: age-related management and outcomes reported in the German Registry for Acute Aortic Dissection Type A(GERAADA) of over 2000 patients. Ann Surg. 2014;259(3):598-604.

13. Nienaber CA, Powell JT. Management of acute aortic syndromes. Eur Heart J. 2012;33:26-35b.

14. Meszaros I, Morocz J, Szlavi J, et al. Epidemiology and clinicopathology of aortic dissection. Chest. 2000;117:1271-278.

15. Erbel R, Aboyans V, Boileau C, et al. 2014 ESC Guidelines on the diagnosis and treatment of aortic diseases: document covering acute and chronic aortic diseases of the thoracic and abdominal aorta of the adult. The Task Force for the Diagnosis and Treatment of Aortic Diseases of the European Society of Cardiology (ESC). Eur Heart J. 2014;35(41):2873-926. 
17. DeBakey ME, Beall AC Jr, Cooley DA, Crawford ES, Morris GC Jr, Garrett HE, Howell JF. Dissecting aneurysms of the aorta. Surg Clin North Am. 1966;46: 1045-55.

18. Yamamoto H, Kadohama T, Yamaura G, et al. Total arch repair with frozen elephant trunk using the "zone 0 arch repair" strategy for type A acute aortic dissection. J Thorac Cardiovasc Surg 2019; 159:36-45.

19. Goldfinger JZ, Halperin JL, Marin ML, Stewart AS, Eagle KA, Fuster V. Thoracic aortic aneurysm and dissection. J Am Coll Cardiol. 2014;64:1725-39.

20. Kawahito K, Adachi H, Murata S, Yamaguchi A, Ino T. Coronary malperfusion due to type A aortic dissection: mechanism and surgical management. Ann Thorac Surg. 2003;76:1471-76.

21. Karck M, Chavan A, Hagl C, et al. The frozen elephant trunk technique: a new treatment for thoracic aortic aneurysms. J Thorac Cardiovasc Surg. 2003; 125:1550-53.

22. Heinz Jakob, Mohammed Idhrees, Mohamad Bashir. Frozen elephant trunk with straight vascular prosthesis. Ann Cardiothorac Surg 2020;9(3):164-169 http://dx.doi.org/10.21037/acs-2020-fet-60

23. Nishida H, Tabata M, Fukui T, Sato Y, Kin H, Takanashi S. A systematic approach to improve the outcomes of type A aortic dissection. J Thorac Cardiovasc Surg. 2017;154:89-96.
24. Van Poucke S, Stevens K, Marcus AE, et al. Hypothermia: effects on platelet function and hemostasis. Thromb J. 2014;12(1):31.

25. Zindovic I, Sjogren J, Ahlsson A, et al. Recombinant factor VIIa use in acute type A aortic dissection repair: a multicenter propensity-score-matched report from the Nordic Consortium for Acute Type A Aortic Dissection. J Thorac Cardiovasc Surg. 2017;154(6):1852e2-1859e2.

26. Zindovic I, Gudbjartsson T, Ahlsson A, et al. Malperfusion in acute type A aortic dissection: an update from the Nordic Consortium for Acute Type A Aortic Dissection. J Thorac Cardiovasc Surg. 2019;157:1324.e6-1336.e6.

27. Di Bartolomeo R, Pantaleo A, Berretta P, Murana G, Castrovinci S, Cefarelli M, Folesani G, Di Eusanio M. Frozen elephant trunk surgery in acute aortic dissection. J Thorac Cardiovasc Surg. 2015 Feb;149(2 Suppl):S105-9.doi: 10.1016/j.jtcvs.2014.07.098. Epub 2014 Aug 10. PMID: 25212056.

28. Sun L, Qi R, Chang Q, et al. Surgery for marfan patients with acute type a dissection using a stented elephant trunk procedure. Ann Thorac Surg. 2008;86(6):1821-25. doi:10.1016/j.athoracsur. 2008.08.026 